Results Respondents in the most deprived neighbourhoods undertook a greater proportion of their MVPA in and around their home location, in buildings, on the road network and in shopping areas compared to respondents from the most affluent neighbourhoods. The proportion of MVPA undertaken in green space was considerably higher in the most affluent compared to the most deprived neighbourhoods (28.9\% vs. $8.8 \%$ ). The places where people undertake physical activity vary according to neighbourhood deprivation suggesting differences in the purpose of activity and access to places for physical activity.

Conclusion Understanding where people undertake their physical activity provides valuable insights into the types of land uses that are important for physical activity and how they vary according to an area based indicator of socio-economic status.

\section{OP16 HOW IS OBJECTIVELY MEASURED PHYSICAL ACTIVITY ASSOCIATED WITH FALLS AND FEAR OF FALLING IN OLDER COMMUNITY DWELLING MEN?}

doi:10.1136/jech-2012-201753.016

'BJ Jefferis, 'S Iliffe, ${ }^{3} \mathrm{~N}$ Kerse, ${ }^{2} \mathrm{D}$ Kendrick, ${ }^{4} \mathrm{~S}$ Trost, 'LT Lennon, 'S Ash, 'SG Wannamethee, 'R Morris, ${ }^{5} \mathrm{PH}$ Whincup. 'Primary Care \& Population Health, UCL, London, UK; ${ }^{2}$ Division of Primary Care, University of Nottingham, Nottingham, UK; ${ }^{3}$ Department of General Practice and Primary Health Care, University of Auckland, Auckland, New Zealand; ${ }^{4}$ Department of Nutrition and Exercise Sciences, Oregon State University, Corvallis, OR, USA; ${ }^{5}$ Division of Population Health Sciences and Education, St George's University of London, London, UK

Background Falls are a major cause of disability in older age and fear of falling (FOF) may limit physical activity. Associations between falls, fear of falling and objectively measured physical activity (PA) has been little studied, particularly in communitydwelling older adults.

Methods The study sample was men participating in a prospective, population-based cohort study in 24 British towns provided questionnaire information on a history of falls in the previous year, fear of falling, and other medical history and wore an Actigraph GT3X accelerometer for 7 days. Uniaxial accelerometer data were analysed in 60 s epochs, excluding bouts of $>60$ minutes of complete inactivity. Days with $>=600$ minutes valid wear time were included. PA intensity was categorised as sedentary: $<100$ counts/minute, light: 100-1952 counts/minute and moderate to vigorous PA (MVPA):>1952 counts/minute. Associations between activity levels and falls, or fear of falling, were estimated using hierarchical linear regression models accounting for clustering and controlling for confounders.

Results A total of 1543 men with a mean age of 77(range 71-91y) wore the Actigraph; 1100 (72\%) had $>600$ minutes wear time on 5-7 days and questionnaire data. $21 \%(n=229)$ had fallen, $30 \%(n=68)$ received medical attention. Men who fell took fewer steps/day than men who did not: mean difference (adjusted for age, day order, month, wear time and town) $-504(95 \% \mathrm{CI}-216,-792)$ and spent more minutes sedentary $13(95 \% \mathrm{CI} 4,22)$, and less in light $-10(95 \% \mathrm{CI}-1,-19)$ and MVPA $-3.5(95 \% \mathrm{CI}-1.3,-5.8)$. In relative terms, the reduction in MVPA was greatest: $22 \%(95 \%$ CI 7,37$)$. Differences were explained by exercise self-efficacy and mobility limitations, but not by exercise outcome expectation. 13\% ( $n=141)$ men reported FOF, of whom $53 \%(n=74)$ had fallen in the past year. Men with FOF took markedly fewer steps than men without:$1325(95 \%$ CI $-1646,-1005)$, spent more minutes in sedentary $29(95 \% 18,40)$, and less in light activity $-21(95 \%$ CI $-32,-11)$ or MVPA $-9(95 \%$ CI $-11,-7)$. In relative terms, the reduction in MVPA was greatest:-72\%(95\%CI -93,-52). Differences were partly explained by exercise self-efficacy, mobility limitations, and to a lesser extent, exercise outcome expectation. There was no evidence that FOF had a greater impact on PA levels among men who had fallen compared to those who had not fallen.
Conclusion PA levels and in particular step counts and MVPA levels, were lower among men who had fallen in the past year, and even more markedly, among those who reported FOF.

\section{Mental Health I}

\section{OP17 DO LOW LEVELS OF PSYCHOLOGICAL DISTRESS PREDICT MORTALITY? EVIDENCE FROM AN INDIVIDUAL PARTICIPANT META-ANALYSIS OF TEN PROSPECTIVE COHORT STUDIES}

doi:10.1136/jech-2012-201753.017

${ }^{1-3} \mathrm{TC}$ Russ, ${ }^{4} \mathrm{E}$ Stamatakis, ${ }^{4} \mathrm{M}$ Hamer, ${ }^{1-3,5} \mathrm{JM}$ Starr, ${ }^{4} \mathrm{M}$ Kivimaki, ${ }^{3,4} \mathrm{GD}$ Batty. ${ }^{1}$ Scottish Dementia Clinical Research Network, NHS UK, Perth, UK; ${ }^{2}$ Alzheimer UK Dementia Research Centre, University of Edinburgh, Edinburgh, UK; ${ }^{3}$ Centre for Cognitive Ageing \& Cognitive Epidemiology, University of Edinburgh, Edinburgh, UK; ${ }^{4}$ Department of Epidemiology \& Public Health, UCL, London, UK; ${ }^{5}$ NHS Lothian, Edinburgh, UK

Background A series of studies have linked psychological distress (depression and anxiety) with cause-specific mortality, but the risk of lower, sub-clinically symptomatic, levels of distress - hitherto of little interest to specialist mental health practitioners - has not been quantified.

Methods We conducted an individual participant meta-analysis of ten large prospective cohort studies from the Health Survey for England. Baseline psychological distress was measured using the 12-item General Health Questionnaire, and mortality from death certification. The analytical sample comprised 68,222 individuals from general population samples of adults aged 35 and over, free of cardiovascular disease and cancer and living in private households in England at study baseline. The main outcome measures were death from all causes $(n=8,365)$, cardiovascular disease $(n=3,382)$, all cancers $(n=2,552)$, and external causes $(n=386)$. Mean (standard deviation) follow-up was 8.2 (3.5) years.

Results There was a positive, dose-response association between psychological distress across the full range of severity and mortality risk (age- and sex-adjusted hazard ratio for General Health Questionnaire scores of $1-3$ compared to zero: $1.20,95 \%$ CI 1.13 to 1.27 ; scores 4-6: 1.431 .31 to 1.56 ; and scores $7-12$ : $1.94,1.66$ to 2.26 ; $\mathrm{p}$ for trend $<0.001)$. This association remained after adjustment for somatic co-morbidity plus behavioural and socioeconomic factors. A similar association was found for death from cardiovascular disease, cancer, and external causes.

Conclusion Psychological distress is associated with increased risk of mortality from several major causes in a dose-response pattern. Even at lower levels of distress the mortality risk was elevated.

\section{OP18 CHILDLESSNESS IN EUROPE: IMPLICATIONS FOR WELLBEING IN LATER LIFE}

doi:10.1136/jech-2012-201753.018

1.2S Gibney. ${ }^{1}$ Centre for Behaviour and Health, Geary Institute, University College Dublin, Dublin, Ireland; ${ }^{2}$ School of Public Health, Physiotherapy and Population Science, University College Dublin, Dublin, Ireland

Background In addition to low patterns of fertility experienced in the last 15 years, lifetime childlessness is increasing in Europe. It is now expected that between 10-20\% of women born in the 1950s in Western Europe will never have children. Adult children play an important supportive role to their ageing parents; economically, acting as bridges to social support and monitoring health behaviour. This role has been enshrined at various institutional levels, from the family to the welfare state. Explanations of the negative relationship between childlessness and late life wellbeing have mainly focused on social support deficits. However, contradictory evidence of the effect of childlessness on psychological wellbeing exists which may be dependent on the wellbeing measures employed, the life course stage under 